\title{
Sprachverwendung in der Politik und ihre Funktionen Eine Forschung \\ vorgelegt von
}

\author{
Magi Mohamad Samir
}

Oberassistentin an der Abteilung für Germanistik

Sprachenfakultät (Al-Alsun); Minia Universität

\section{Inhaltsverzeichnis}

\section{Einleitung}

1. Rolle der Rezipienten im Politikprozess

2. Funktionen der Sprache in der Politik

3. Schlussfolgerung

-Literaturverzeichnis

-Internet-Quellen

\section{Einleitung}

Während die Sprache in der Tat das mächtigste Instrument menschlicher Kommunikation ist, sind Sprache, Kommunikation und Rede das originäre Instrument der Politik. Die Politiker müssen in den meisten Fällen die Öffentlichkeit informieren, politisches Handeln begründen, analysieren, kritisieren und rechtfertigen. Sie bewerten bestimmte Sachverhalte positiv oder negativ, stützen die eigene Position argumentativ und werten den Gegner ab. ,Politiker appellieren an die Emotionen ihres Publikums, sie buhlen um seine Zustimmung und sie werben für ihre Überzeugungen. Dies geschieht im Wesentlichen in und durch Sprache. Sprache ist nicht nur irgendein Instrument der Politik, sondern überhaupt erst die Bedingung ihrer Möglichkeit. "'

Dieckmann bestimmt die Politik als ,,staatliches oder auf Staat bezogenes Reden “. Diese Definition zeigt einen kommunikationsorientierten Hinweis auf den Zusammenhang von Sprachverwendung und Politik. Eine leistungsfähigere Definition von Politik findet sich bei Strauß u.a., der Politik als ,in sich differenzierten Großbereich der Kommunikation, in dem über Angelegenheiten öffentlichen Interesses gehandelt wird, in dem Meinungen gefaßt werden und Prozesse ablaufen, die der Herstellung und Durchsetzung verbindlicher oder auch umstrittner gesellschaftlicher Entscheidungen dienen." bezeichnet. ${ }^{3}$ Heringer spricht von Politik in der Sprache anstatt von Sprache in der Politik: „Politik in Sprache soll darauf hinweisen, daß Politik sich in Sprache vollzieht, daß politische Tätigkeit sprachliche Tätigkeit ist. " 


\section{Rolle der Rezipienten im Politikprozess}

Beim Sprechen über Politik wird die Rolle des Kommunikators von Bürgern ausgefüllt. Es handelt sich um private bis halböffentliche Gespräche und Diskussionen über Politik, in denen die Teilnehmer aktiv am politischen Meinungsbildungsprozess teilnehmen dürfen. Bei der politischen Mediensprache hingegen übernehmen Journalisten die Rolle des Kommunikators, z.B. Tageszeitungen, politische Magazine oder Nachrichtensendungen. Der Bürger als Rezipient hat jedoch eine deutlich passive Rolle. Bei der Politikersprache stellen die Politiker selbst die Kommunikatoren dar. Hier handelt es sich also um öffentlichpolitisches Sprechen in persuasiver Funktion. Es lassen sich je nach RezipientenGruppe und -Rolle zwei sprachliche Kategorien unterscheiden: der Bereich der politischen Binnenkommunikation: Sprache in der Politik (andere Politiker als Rezipienten) und der Bereich der politischen Außenkommunikation: Politikersprache / Sprache der Gesetzgebung (Bürger bzw. Journalisten als Rezipienten). Die politische Außenkommunikation lässt sich noch einmal unterteilen in die Politikersprache sowie die Sprache der Gesetzgebung. Während sich die Politikersprache hierbei (ähnlich der politischen Mediensprache) an den Bürger als Subjekt der öffentlich-politischen Willensbildung richtet, erscheint der Bürger im Rahmen der Sprache der Gesetzgebung als Objekt, das sich an bestimmte Regeln zu halten hat. Den vierten Bereich der politischen Sprache bildet die Sprache der Verwaltung und der Rechtsprechung. Ihre Kommunikatoren sind nicht-gewählte Beamte bzw. Richter, und ihre Adressierung richtet sich an einzelne Bürger.

\section{Funktionen der Sprache in der Politik}

Aus der oben dargestellten Meinung ergibt sich, dass je nachdem, welche Kommunikatoren und welche Rezipienten am jeweiligen Politikprozess beteiligt sind und welche Rolle sie dabei einnehmen, sich auch spezifische Funktionen und Merkmale der jeweiligen politischen Sprache feststellen lassen. Politiker bewegen sich demnach in verschiedenen Sprachfeldern, Sprachebenen und Sprachstilen. Die Politikersprache ist von zwei Funktionen charakterisiert; nämlich der Persuasionsfunktion und der Integrationsfunktion. Der Prozess der öffentlichpolitischen Willensbildung kann als ständiges Ringen von Politikern verstanden werden, um die Zustimmung der Bevölkerung zu erreichen. Daher ist die politische Sprache als emotiv, symbolhaft und werthaft gekennzeichnet. Eng mit dieser Charakterisierung verbunden ist die Einstufung der Politikersprache als eine Sprache der Schlagwörter. Begriffe wie „Freiheit", ,Gerechtigkeit", „Solidarität“, „Recht“ u.a. überdecken aufgrund ihrer großen Bedeutungsbandbreite eine Vielzahl von unterschiedlichen und manchmal widersprüchlichen Meinungen, sind jedoch gleichzeitig hochgradig emotional aufgeladen. Meistens wird in den politischen Reden von „Herausforderung“ statt von „Problem“ gesprochen, weil der Begriff „Herausforderung“ eine positive Grundhaltung impliziert. Ein „Problem“ ist eine Hürde, während eine „Herausforderung“ eine Chance darstellt. 


\section{Schlussfolgerung}

McGuire beschreibt 1989 die Wirkung von kommunikativen Überzeugungsversuchen als mehrstufigen Persuasionsprozess, der mindestens fünf Schritte umfasst: Aufmerksamkeit, Verständnis, Zustimmung, Speicherung und Handlung.5 Wirksamkeit und Verständlichkeit von politischer Sprache kann in einem nicht eindeutigen bis widersprüchlichen Verhältnis stehen. Im Gegensatz zu Alltagskommunikation stehen oft bei der politischen Kommunikation Verständigungsprobleme zwischen Politikern und Bürgern. Diese Probleme werden zudem durch die massenmediale Übertragung und die Einseitigkeit der Kommunikation verstärkt.

1Girnth, Heiko (2002): Sprache und Sprachverwendung in der Politik. Eine Einführung in die linguistische Analyse öffentlich-politischer Kommunikation, Tübingen: Max Niemeyer, S. 1

2Dieckmann, Walther (1975): Sprache in der Politik. Einführung in die Pragmatik und Semantik der politischen Sprache. Heidelberg: Sprachwissenschaftliche Studienbücher Abt. 2, S. 29

3Strauß, Gerhard; Haß, Ulrike; Harras, Gisela (1989): Brisante Wörter von Agitation bis Zeitgeist. Ein Lexikon zum öffentlichen Sprachgebrauch. Berlin / New York: de Gruyter, S. 29

4Heringer, Hans Jürgen (1990): „Ich gebe Ihnen mein Ehrenwort“ Politik-Sprache-Moral. München: C. H. Beck, S. 9

5Vgl. McGuire, William J. (1989): Theoretical Foundations of Campaigns. In: Rice, Ronald E. ; Atkins, Charles K. (Hrsg.): Public Communication Campaigns. 2. Auflage. Newbury Park: Sage, S.44 ff. 